\title{
Legal Shifts in the Process of Proof
}

\author{
Floris Bex \\ Argumentation Research Group \\ University of Dundee \\ Dundee, UK \\ florisbex@dundee.ac.uk
}

\author{
Bart Verheij \\ Department of Artificial Intelligence \\ University of Groningen \\ Groningen, The Netherlands \\ b.verheij@ai.rug.nl
}

\begin{abstract}
In this paper, we continue our research on a hybrid narrativeargumentative approach to evidential reasoning in the law by showing the interaction between factual reasoning and legal reasoning. We therefore emphasize the role of legal story schemes (as opposed to factual story schemes that formed the heart of our previous proposal). Legal story schemes steer what needs to be proven, but are also selected on the basis of what can be proven. They provide a coherent, holistic legal perspective on a criminal case that steers investigation and decision making. We present an extension of our previously proposed hybrid theory of reasoning with evidence, by making the connection with reasoning towards legal consequences. We discuss the phenomenon of legal shifts that shows that the step from evidence to (proven) facts cannot be isolated from the step from proven facts to legal consequences. We show how legal shifts can be modelled in terms of legal story schemes. Our model is illustrated by a discussion of the Dutch Wamel murder case.
\end{abstract}

\section{Keywords}

Legal argumentation, Legal evidence and proof, Stories

\section{INTRODUCTION}

In research on legal reasoning in the field of AI \& law, models of reasoning from proven facts to legal consequences are well developed (e.g., [22], [13]). Recently there is also a rise in attention for evidential reasoning (e.g. [5], [17], [25]). However, there is not much recent work that aims at modelling the full pathway from evidence via proven facts to legal consequences (except maybe [27], see section 5). Providing a model of that full pathway is the focus of his paper.

We will argue that the factual and legal part of legal reasoning (the steps from evidence to proven facts, and from proven facts to legal consequences, respectively; cf. Figure 1) cannot be separated. The phenomenon of legal shifts is a case in point: the legal perspective on a case changes as a result of what can be proven, and at the same time determines the investigative and decision making focus. We provide an example based on the Dutch case of a murder in the provincial town of Wamel (as carefully analyzed by Israëls [16]). In this case, it was impossible to successfully charge the prime suspect for murder, but he is successfully charged (and convicted, after appeal and withstanding cassation by the Supreme Court) as an accomplice to

Permission to make digital or hard copies of all or part of this work for personal or classroom use is granted without fee provided that copies are not made or distributed for profit or commercial advantage and that copies bear this notice and the full citation on the first page. To copy otherwise, or republish, to post on servers or to redistribute to lists, requires prior specific permission and/or a fee.

ICAIL '11, June 6-10, 2011, Pittsburgh, PA

Copyright @ 2011 ACM 978-1-4503-0755-0/11/06 ... \$10.00 the murder, while the key question of who shot the gun is not really clarified.

We will extend our previous work on reasoning with evidence ([2], [4], [6]), in which we mainly concerned ourselves with the factual part of trials, that is, the evidence and the facts which we might infer through evidential reasoning. As of yet we have not specified how exactly, once the facts have been established, the reasoning towards the legal consequences proceeds. Also, the exact way in which the law might influence the reasoning about the facts and the evidence is an open question.

The contribution of the paper is as follows. We propose an extension of our hybrid narrative-argumentative approach to evidential reasoning ([2], [4], [6]) in order to incorporate the complete reasoning process in a case: from evidence via facts to legal consequences. The focus is on analytic tools for the stages of this process. Our proposal allows a representation of the mentioned phenomenon of legal shifts, in which one legal perspective is abandoned favouring another. For that purpose, the additional modelling tool of legal story schemes, which can be used to establish the legal coherence (as opposed to the factual coherence) of a case, is required. ${ }^{1}$

Factual and legal story schemes make explicit that a holistic, coherent perspective underlies all reasoning and argumentation. Factual story schemes are important to determine which accounts of the facts are plausible, and which gaps need to be filled by further evidence in order to form a full picture of what happened. Legal story schemes show which sets of (legally qualified) facts and events are recognized as coherent wholes by the law, in the sense that a legal consequence such as a conviction can be based on them. We will show how both factual and legal story schemes are a tool to reduce the risk of tunnel vision.

In the following, we start with a discussion of the theoretical background used (section 2). This comprises a discussion of the theory construction view on legal reasoning on the basis of evidence via facts to legal consequences. We also provide a brief overview of the Wamel murder case that we use as an illustration. Then follows a review of the evidential part of our hybrid theory (section 3). In section 4, the hybrid theory is extended to include the legal step in the pathway from evidence via facts to legal consequences. In section 5 , we put our approach in perspective by a discussion in the light of related research. We conclude our paper in section 6 , pointing out some issues for future research.

\section{FROM EVIDENCE VIA FACTS TO LEGAL CONSEQUENCES (AND BACK)}

Reasoning in a criminal case is a complex process in which the available evidence determines which facts can be proven, and hence also which legal consequences follow. As we already

\footnotetext{
${ }^{1}$ There exist other formal approaches to coherence, e.g. [14], [2].
} 
indicated above, this also works the other way: the law often regulates which facts have to be proven, which in turn determines which of the evidence is relevant. As a result, a theory construction perspective, in which the overall reasoning about a legal case (from evidence, via facts to legal consequences) is gradually constructed is in place (Figure 1, adapted from Verheij 2005 , p. 10). The figure not only suggests that evidence, facts and legal consequences are gradually constructed from an imperfect initial version, but also there is an influence both upward (from evidence via proven facts to legal consequences) and downward (from legal consequences via proven facts to evidence).

Reasoning in a criminal case is a complex process in which the available evidence constrains which facts can be proven, which in turn are the grounds for legal consequences on the basis of the applicable legal rules (Figure 1, adapted from [26], cf. also [18]). The path of justification is from evidence via facts to legal consequences; in the figure, this is visualised as the white upward arrows. However, there is also an influence in the other direction. This influence is not about justification but rather has to do with the discovery or exploration of new facts and evidence: the legal perspective on a case (in the form of the legal consequences that are at issue) steers which facts need to be proven for the legal consequences to actually follow, while the facts that have to be proven guide the search for evidence.

To sum up, we view legal (evidential) reasoning from a theory construction perspective. Figure 1 not only suggests that evidence, facts and legal consequences are gradually constructed from an imperfect initial version, but also that there is an influence both upward (from evidence via facts to legal consequences) and downward (from legal consequences via facts to evidence).

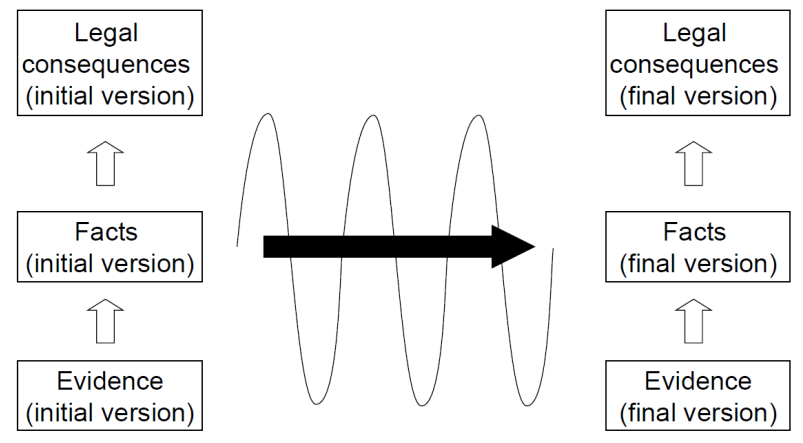

Figure 1: A theory construction perspective on legal reasoning

As for terminology, with evidence we mean the evidential data, the primary sources of evidence the existence of which cannot be sensibly denied (e.g. witness statements made in court, forensic expert reports handed to the jury). As is usual in law, we use the term fact to denote descriptions of states or events, the truth of which are currently unknown and have to be proven (cf. the terms "fact-finder", "question of fact"). ${ }^{2}$ By legal consequences, we mean the legal states of affairs that the law attaches to the facts

${ }^{2}$ We assume a rough distinction between "real-world" facts, which tell us something about the (physical) reality (e.g. Francis was angry with Kevin, Francis shot Kevin) and legal facts, facts which tell us something about the legal reality (e.g. Francis' killing of Kevin was premeditated, Francis is punishable for murder). (e.g. the punishability for murder and the ensuing sentence of imprisonment; cf. the German jurisprudential term Rechtsfolge).

In previous work ([2], [4], [6]) we focused on the bottom part of Figure 1, that is, evidential reasoning about the evidence and the (real-world) facts. We argue that this evidential reasoning is best modelled in a hybrid theory that uses both stories and arguments. In the hybrid theory, facts are organised into multiple hypothetical stories, coherent accounts of what (might have) happened in the case. Arguments based on evidence can then be used to justify these stories, as these arguments can be used to support elements in a story with evidence or, in other words, to anchor the story in the evidence (cf. [28]). Ultimately, the alternative stories in a case should be compared and the best one (i.e. the most coherent one that best fits the evidence) should be chosen.

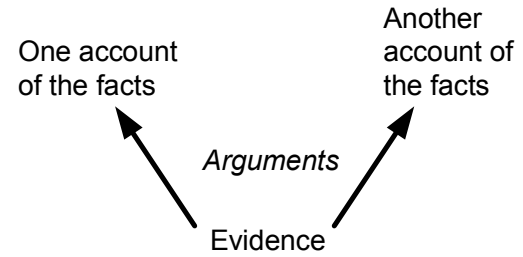

Figure 2: Hybrid narrative-argumentative evidential reasoning

Figure 2 visualises the basic idea behind the hybrid theory. This way of hybrid reasoning with stories and arguments is particularly close to the analytic and evaluative thinking of the professionals that actually perform the reasoning in a criminal case, in particular criminal investigators and judges ([18], [28], [2]).

At first view, legal reasoning - reasoning about the (legal) facts and legal consequences (the top part of Figure 1) - seems quite different from evidential reasoning (cf. the dichotomy between a "question of fact" and a "question of law" and Wigmore's separation of factual proof and legal admissibility in evidential reasoning). Whilst the content and context of evidential reasoning are different from those of legal reasoning, the reasoning mechanisms employed are related.

In legal reasoning, the legal consequences are organised into clusters of legal facts that provide legally coherent perspectives on a case. In our opinion, such a legally coherent perspective can be seen as a "legal story" about what happened in the case, a legally qualified account of what happened. This is particularly apparent in Dutch cases, where the indictment is usually phrased as a small legal story (intermixed with their legal qualification). For example, a murder charge in our case could read as follows: "On the $5^{\text {th }}$ of January 1997, Francis intentionally and with malice aforethought killed Kevin by shooting him repeatedly with a semiautomatic weapon, which caused multiple severe injuries to Kevin, as a result of which Kevin died".

In any case, there are at least two legal accounts representing the "guilty" and "innocent" perspectives. ${ }^{3}$ Arguments can be used to reason from the (real-world) facts to the legal consequences, that is, to justify the legal accounts with the facts of the case which are themselves justified by the evidence. Ultimately, the alternative legal accounts should be compared and the best one (i.e. the one that best fits the proven facts) should be chosen. Figure 3 shows

\footnotetext{
${ }^{3}$ In practice, there might be more alternative legal accounts in a case and the alternatives will be more specific.
} 
how legal accounts follow from the facts of the case in a similar way as the facts follow from the evidence in the case.

Now, the combination of evidential reasoning (Figure 2) and legal reasoning (Figure 3) gives us all the machinery we need for the reasoning in a criminal case (Figure 1). Because of the similarities between evidential and legal reasoning, we think that this combination can be modelled without introducing too much new conceptual and logical machinery. However, the exact connection and similarities between the evidential and the legal layer will have to be made more clear, particularly if we not only consider the upward, justificatory influence of evidence on legal consequences but also the downward, exploratory influence of legal consequences on evidence.

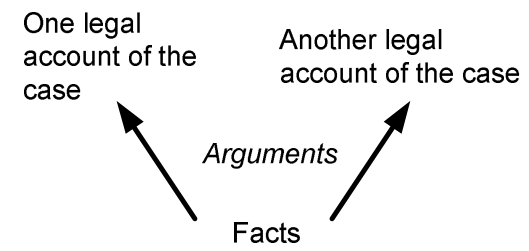

Figure 3: Hybrid narrative-argumentative legal reasoning

\subsection{The Wamel case}

Below we will briefly discuss the key concepts of stories and arguments. We will illustrate these concepts using examples inspired by the Wamel case [16], which also serves as a running example throughout the paper. In the Wamel case, there are three key actors: Kevin Moyson, the victim, Sander Mornie, the main witness and friend of Kevin's, and Francis Liebrand, the prime suspect and an acquaintance of Kevin's. All three are members of a loosely connected community of petty criminals often related to soft drugs and theft, but not normally associated with big organized crime.

On January 6, 1997, Kevin's body is found some 50 kilometres from his home town Uden, near two barns in the village of Wamel. He has been shot dead. Later that day, Sander contacts the police and states that he was also at the scene of the crime, allegedly trying to escape. According to Sander's initial, later denied, statements, Francis was also at the barns and an argument developed between Kevin and Francis (allegedly over a 5000 guilders debt that Francis owed Kevin). Francis then walked to the back of one of the barns. When Kevin followed him, there was a sudden firing of shots, after which Sander fled.

Francis was ultimately convicted both in first and second instance to 13 years imprisonment, as an accomplice to the murder of Kevin and the attempted murder of Sander. ${ }^{4}$ This was later confirmed by failing cassation at the Supreme Court.

\footnotetext{
${ }^{4}$ In this paper, we generally assume a Dutch legal framework. Even though the use of legal terms in different jurisdictions has many pitfalls, such terms will be translated more or less literally and where necessary we will briefly sketch the legal context. For example, a relevant distinction here is that the principal is the primary actor of a crime and the accomplice an active participant.
}

\section{EVIDENTIAL REASONING IN THE HYBRID THEORY}

The hybrid theory is essentially a theory of Inference to the Best Explanation (IBE). Given a set of facts that have to be explained, we hypothesize various causally connected stories from which these facts follow. We then use arguments based on evidence to reason about these stories in order to determine which story is the best explanation (i.e. the one that is best supported by evidence). Thus, the formal hybrid theory is a combination of a causal theory for building hypothetical stories about the facts, and an evidential theory for building arguments from the evidence to the facts. More specifically, the formal hybrid theory is a combination of a formal model of causal-abductive reasoning $C T$, which is based on model-based approaches to causal-abductive inference (e.g. [10]) and an evidential argumentation theory ET, which takes its inspiration from standard accounts of structured and abstract argumentation ([22], [20], [11]).

\subsection{Abductive reasoning with stories and story schemes}

A criminal case usually starts when some initial evidence is found that points to the possibility that a crime has been committed. In the Wamel case, the investigation started with Sander contacting the police and telling them that Kevin had been shot near the barns in Wamel; Kevin's body was found the same day near the barns. On the basis of this preliminary evidence, we can formulate one or more initial explananda, facts that have to be explained. These explananda can be observations or they can be other facts that are considered important (this will be further discussed in section 4.3 when we look at the connection to the legal reasoning layer). In the Wamel case, the most important initial explanandum is the fact that Kevin died due to multiple gunshot wounds.

Explananda can be explained by constructing stories - coherent sequences of states and events - about what might have happened. A coherent story (cf. [18], [25]) is causally connected in that the individual events are connected by (sometimes implicit) causal links. This allows us to perform abductive reasoning in order to infer what happened to Kevin. Abductive reasoning involves "guessing" new hypotheses that explain some data (i.e. Kevin's body). The basic idea of such reasoning is that if we have a general rule ' $c$ is a cause for $e$ ' and we observe $e$, we are allowed to infer $c$ as a possible hypothetical explanation of the effect $e$. This cause $c$ which is used to explain the effect can be a single state or event, but it can also be a causally connected chain of events, a story. One explanation for Kevin's death is that Sander and Kevin went to the barns in Wamel to meet Francis and that Francis shot Kevin, which caused Kevin's death.

In our formal model, abductive reasoning is done using the causal theory $C T=\left(R_{\mathrm{C}}, H, F\right)$, where $R_{\mathrm{C}}$ is a set of causal rules, $H$ is a set of hypothetical events (ground literals) and $F$ is the set of explananda (ground literals). Causal rules in $R_{\mathrm{C}}$ are formalised using a connective for defeasible implication $\Rightarrow c$, which only satisfies the inference rule for (defeasible) modus ponens. A specific causal relation between events can then be formalised as a rule, e.g. Francis shot Kevin $\Rightarrow c$ Kevin died. As usual, a rule with variables is a scheme standing for all its ground instances. Thus, a specific causal link can be generalized as a causal generalization, viz. $r_{\mathrm{c} 1}: x$ shoots $y \Rightarrow c y$ dies.

Given these causal rules and the set of hypothetical events $H$, we can build a story. In $C T$, a story is then a finite sequence of events 
and rules expressing the explicit causal relations in the story. The ordering of the events suggests their chronological succession. Individual events in the story are then hypothetical events from $H$ or events which are causally inferred from other events in the story by applying defeasible modus ponens to these other events and a rule from $R_{\mathrm{C}}$. Furthermore, as is usual in abductive-causal models, we enforce that a story be consistent by requiring that $S$ $H \mathrm{CA} \perp$. Stories $(C T)$ denotes the set of all stories that can be constructed from a theory $C T$. As an example, consider the story $S_{1}$ :

[Kevin was at the barns, Francis was at the barns, Francis shot Kevin, Francis shot Kevin $\Rightarrow$ C Kevin died, Kevin died].

Notice that in order to provide a more holistic flavour to the causal theory, not all causal relations in a story will be made explicit: the causal coherence of a story then depends on implicit causal relations, which will be discussed below when we look at story schemes.

In the causal theory, the "explains" relation between a story and the explananda is defined through a notion of logical consequence. That is, a story $S$ explains an explanandum $f$ iff $S \vdash c$ $f$, where $\vdash_{c}$ stands for logical consequence according to the set of all deductive inference rules extended with modus ponens for $\Rightarrow c$. This means that the explanandum is either a hypothetical event in $S$ or can be derived from previous events in $S$ using causal rules. For our initial explanandum Kevin died, we can abductively infer $S_{1}$ as an explanation, as Kevin died follows from $S_{1}$.

Note that using causal rules, we can also perform predictive reasoning, where one assumes a hypothesis and tries to predict what will be the case as a consequence of this hypothesis. For example, if we hypothesize that Francis shot Kevin, there should be (Kevin's) blood at the scene of the crime, as we would normally expect that being shot causes one to bleed quite heavily. This predictive reasoning can then be used in the search for new evidence (section 3.3).

In our earlier work [6], explicit causal rules played a larger role. Following standard accounts of logical causal-abductive reasoning, the explanatory stories in the previous version of the hybrid theory were represented as fully connected causal networks. While certain explicit causal generalizations play an important role in causal-abductive reasoning, they are atomistic in flavour. Explicit causal relations are used to link small clusters of facts and events, these clusters are considered separately and the case is not considered 'as a whole'.

In contrast, a holistic perspective on story coherence is provided by story schemes, abstract scenarios that can serve as a scheme for particular stories. As is argued by [24], the knowledge that is used when thinking about stories often does not have the form of individual causal generalizations but is more naturally thought of as a collection of generalized events or event types, abstract renditions of how things generally happen in the world. One example of such a story scheme is [18]'s story scheme for intentional actions: given some initial states of affairs, a motive may lead to an action with certain consequences.

For our formal definition of story schemes we basically follow the definitions given in [2]. We assume a set FSS containing the relevant factual story schemes in $C T .^{5}$ Because story schemes are in effect abstract stories, the structure of story schemes is very close to that of specific stories. Basically, a story scheme $G$ is a sequence containing literal schemes (literals with variables that act as schemes for all their ground instances) that denote event types and causal rules that denote types of causal links. As with stories, the sequence represents a basic idea of chronological succession.

As an example of a story scheme, take the simple scheme for murder (in a non-legal sense):

[ $x$ is at place $p, y$ is at place $p, x$ has motive $m$ to kill $y, x$ shoots $y$, $x$ shoots $y \Rightarrow c y$ dies, $y$ dies].

Note that there is an explicit causal rule in this scheme, which says that $x$ shooting has to be the cause of $y$ dying (otherwise $y$ would not have been killed by $x$ ). This scheme is a concrete version of the scheme for intentional actions (in the sense that murder is an intentional action). A specific story can now be matched to a story scheme by instantiating the variables in the story scheme with constants in the story. In our example, the story $S_{1}$ is an instance of a motivated killing: both Kevin and Francis were in the same place $p$ (the barns) and Francis shot Kevin, which caused Kevin's death.

Notice that in our example there is an element in the story scheme which is not in the story, namely $x$ has motive $m$ to kill $y$ (which would be Francis has motive $m$ to kill Kevin in the story). Even though the events in the story correspond to some element of the scheme, not all elements of the scheme have a corresponding event in the story. We say that a story $S$ completes a story scheme $G$ iff for every element $g \in G$, there is some $s \in S$ such that $s$ is a ground instance of $g$. In this example, the missing motive makes that the story does not complete the scheme.

The concept of completeness can be used to determine the coherence of a story. Recall that a coherent story is causally connected. In our previous work, this causal connectivity was modelled using individual, explicit causal links. In this paper, however, we define coherence through the use of story schemes. More specifically, we consider a scheme to be coherent if it completes a plausible story scheme. This ensures that the story "has all its parts" (e.g. no murder story with a missing motive, cf. [30]). Furthermore, if we assume that story schemes only present coherent views on how things generally work in the world around us, we accept that by completing a scheme the story adheres to the scheme's implicit causal coherence.

Matching a part of a story to a scheme allows for (abductive and predictive) causal reasoning without explicit causal rules. For example, if we have a scheme $\left[e_{1}, e_{2}, e_{3}\right]$ and we observe $e_{3}$, we can infer $\left[e_{1}, e_{2}\right]$ as a possible abductive explanation of $e_{3}$; in our example, Francis has motive $m$ to kill Kevin can thus be abduced from story $S_{1}$ using the story scheme for murder. Similarly, we may perform predictive reasoning using a story scheme; if we extend the murder scheme to include [...x shoots $y, y$ bleeds heavily...], we can use this extended scheme to infer that Kevin bled heavily. Here, the abductive and predictive inference steps are not justified by an explicit causal connection between the

\footnotetext{
${ }^{5}$ Factual story schemes are schemes about real-world facts. They are different from legal story schemes, which will be discussed in section 4.2.
} 
original hypothesis and the new hypothetical event, but rather by the implicit causal connections in the story scheme.

\subsection{Evidential reasoning with arguments}

In the previous section, we were mainly concerned with constructing hypotheses based on our general knowledge of the world, encoded as either atomistic causal rules or holistic story schemes. In reality, however, the process will also be driven by evidence. Here, arguments play an important role as they can be used to connect the evidence to a story: given some evidence, we can infer conclusions through defeasible argumentative inferences until we arrive at some event in our hypothesised story. Thus, we can support a story with evidence.

Our evidential argumentation theory ET takes its inspiration from[21], which integrates [22] and [20]'s ideas on rule-based argumentation and structured arguments within [11]'s abstract approach. In the theory $E T=\left(R_{\mathrm{E}}, K\right), R_{\mathrm{E}}$ is a set of evidential rules or generalizations and $K$ is a consistent set of evidence, the evidential data. The evidential rules in $R_{\mathrm{E}}$ are represented similarly to the causal rules in $R_{\mathrm{C}}$, with the connective $\Rightarrow_{\mathrm{E}}$ (where the subscript E denotes an "evidential" instead of a "causal" inference). These evidential rules, or evidential generalizations, are used to warrant the inferences from evidence to a conclusion. For example, an inference from a witness testimony to some conclusion is only warranted if we accept that witnesses usually speak the truth. This witness testimony generalization can be represented formally as $r_{\mathrm{e} 1}$ : witness $x$ says " $p$ " $\Rightarrow_{\mathrm{E}} p$.

Now, arguments can be built by taking evidence from $K$ and rules from $R_{\mathrm{E}}$ as premises and chaining applications of defeasible modus ponens into tree-structured arguments, where each node in the tree is thus an element of $K$, a rule from $R_{\mathrm{E}}$ or the result of an application of the defeasible modus ponens to one or more other nodes. Such a structure is very similar to the well-known Wigmore charts from the literature [1]. As an example of a simple argument, consider the following simple argument $A_{1}$ :

1. $e_{1}$ : Sander says "Francis shot Kevin" $(K)$

2. $r_{\mathrm{e} 1}$ : witness $x$ says " $p$ " $\Rightarrow_{\mathrm{E}} p \quad\left(R_{\mathrm{E}}\right)$

\section{Francis shot Kevin $(1,2, D M P)$}

We say that $\operatorname{Args}(E T)$ denotes the set of all arguments that can be constructed from a theory $E T$.

Arguments are a tool to deal with the contradictory evidence that is often available in a case. In the Wamel case, for instance, the main witness Sander is unreliable. He has reported two versions of what happened. In the first report, to his girlfriend and her mother, he says that Francis was at the crime. In the second report, to the police, he denies Francis was there. A careful argumentative analysis can help to find out how to handle such conflicting information.

In our formal model, conflict between arguments is modelled as defeat. An argument $A_{1}$ can defeat another argument $A_{2}$ in essentially two ways (cf. [20]). $A_{1}$ and $A_{2}$ rebut each other if they have an opposite (intermediate) conclusion. $A_{1}$ undercuts $A_{2}$ if there is a conclusion $\neg r_{\mathrm{i}}$ in $A_{1}$ and an application of defeasible modus ponens to $r_{\mathrm{i}}$ in $A_{2}$ (where $r_{\mathrm{i}}$ is the name of a rule in $R_{\mathrm{E}}$ ). Here, $\neg r_{\mathrm{i}}$ generalises the two cases where a rule is invalid (inapplicable in all cases) and where there is an exception to the rule (inapplicable in some cases), cf. [13]. For example, we might argue that Sander is lying because he gave contradictory statements. From Sander is lying we can then infer $\neg r_{\mathrm{e} 1}$ (the witness testimony rule), as the situation in which a witness is lying provides an exception to the generalization represented by $r_{\mathrm{e} 1}$, that witnesses normally speak the truth. The argument about Sander's veracity would then undercut argument $A_{1}$ for Francis shot Kevin.

An advantage of formal models of argumentation is that they can be used to evaluate a particular argument given a complex mass of other evidential arguments. In other words, given a collection of arguments and their binary defeat relations, the dialectical status of the arguments can be determined. Following [21], we assume that our theory for arguments instantiates one of [11]'s semantics. For present purposes, the exact type of semantics is not important and we simply assume that arguments can be justified, which means that they are not defeated by other justified arguments, overruled, which means that they are defeated by other justified arguments, or defensible, which means that they are neither justified nor overruled.

\subsection{Combining stories and arguments in a hybrid theory of IBE}

An important part of our hybrid narrative-argumentative version of Inference to the Best Explanation is the consideration of alternative stories. Although in principle the mere contradiction of the story proposed by the prosecution, e.g. by a solid alibi for the suspect, suffices for the preclusion of a conviction, in most cases there are alternative stories, for each of which there is some support (but not conclusively justifying) and for each of which there is some attack (but not conclusively defeating). Moreover, a serious search and consideration of alternative stories is a good tool to lessen the danger of too narrow tunnel vision.

In the Wamel case, a natural suspect (in addition to Francis) might be Sander. Sander was also at the barns and he could have tried to incriminate Francis because he himself has something to do with Kevin's death. Moreover, Sander gave contradictory testimonies, first stating that Francis was the killer and later saying he does not know who was the shooter; it could be that Sander could be lying because he himself is the killer, viz. [Kevin was at the barns, Sander was at the barns, Sander shot Kevin, Sander shot Kevin $\Rightarrow c$ Kevin died, Kevin died]. Note that this story is almost the same as $S_{1}$, only with Sander as the shooter.

In addition to the search for alternatives, the stories themselves also have to be thoroughly analysed and compared. In this analysis, it is important to look at the extent to which the alternative stories conform to the evidence as well as their plausibility, the extent to which the story conform to our knowledge of the world. In the work on the hybrid theory ([2], [4]), a number of criteria are defined that determine the quality of a particular story. These criteria are mostly defined in the hybrid theory, that is, using a combination of $E T$ and $C T$. Below, we will discuss three criteria which are important for current purposes.

Perhaps the most important criterion to asses stories is evidential support, which denotes the sources of evidence from $K$ that support a story $S$ through a justified argument $A \in \operatorname{Args}_{\mathrm{E}}$ (i.e. $e \in$ $K$ is a premise of $A$ and some $s \in S$ is a conclusion of $A$ ). Here, the element of the story $s$ can be either an event or a causal rule. ${ }^{6}$ In our example, the evidence $e_{1}$ : Sander says "Francis shot Kevin" supports the story $S_{1}$, as $e_{1}$ supports the event in the story Francis

\footnotetext{
${ }^{6}$ This is important, as in criminal cases this sort of evidence is often provided (e.g. a forensic report stating that "the victim's death was caused by a bullet").
} 
shot Kevin through the evidential argument. Note that evidence only supports a story through a justified argument: if the argument is defeated, the evidence does not support the explanation as the link between the evidence and the story is effectively "cut".

Related to evidential support is the concept of evidential gaps, the elements of the story (events or causal links) that are not supported by evidence. In the formal hybrid theory, the gaps in a story $S$ are all elements of $S$ that are not the conclusion of a justified argument. These elements are thus hypothesised events for which there is no direct evidence. Even without direct evidence we may still believe some fact if we consider it in conjunction with other facts. If a fact fits a coherent story that has enough evidential support (where the evidence supports other facts in the story), the circumstances detailed in the story make it more plausible that the hypothesized fact happened. This is the 'gap-filling' function of stories [1]: gaps in the evidence are filled with events that fit the total picture painted by the story.

Evidential gaps also play an important role in the investigative phase, as they point to new avenues of investigation. Given a basic story, we can use abduction or prediction to infer story consequences, events which naturally follow from the story. In section 3.1, we already showed two examples of such story consequences, namely Francis has motive $m$ to kill Kevin and Kevin bled heavily. Now, evidential gaps that have been inferred as story consequences are relevant as they guide the search for new evidence; after inferring that Francis should have a motive, we might want to look for evidence for such a motive. Thus, the reasoning about the facts acts out its influence on the (search for) evidence in the case (i.e. the "downward" influence in Figure 1).

Evidential support and gaps concern the connection (or lack thereof) between the story and the evidence. In a case, we also have to consider the inherent plausibility of a story, that is, whether it conforms to our expectations and knowledge of the world. This plausibility depends on whether a story is consistent and coherent (section 3.1). A story's plausibility plays an important part in guiding the investigative process; the police would not, for example, want to pour all its resources into pursuing a clearly implausibly hypothetical story. However, one has to be wary of the danger of preferring a "good" story (i.e. plausible, coherent) to a "true" story (i.e. supported by strong evidence): [18], [28] show that in some cases, the implausible story is actually better supported by the evidence.

The criteria of evidential support and plausibility can be used for a direct comparison of stories. For example, all other things being equal, a story $S$ can be considered better than a story $S^{\prime}$ if the number of sources of evidence that support $S$ is higher than $S^{\prime}$. Thus, we may determine the best explanation in a case. Whether this explanation then leads to a conviction or acquittal depends on further (legal) factors, such as whether the story about the facts fits the appropriate legal story (sections 2 and 3.3), who has the burden of proof and how high the proof standard is [8].

\section{LEGAL REASONING IN THE EXTENDED HYBRID THEORY}

The basic hybrid theory allows for both reasoning from the evidence to the facts as well as from the facts to the evidence. However, it does not include explicit legal reasoning, that is, the reasoning from the facts to the legal consequences and the influence the (desired) legal consequences may have on the various accounts of the facts. In this section, we extend our hybrid theory in order to include such legal reasoning. This extension takes the form of a legal theory $L T=(L S S, R)$, which contains a set of legal story schemes $L S S$ and a set of rules $R=R_{\mathrm{L}} \cup R_{\mathrm{Q}}$ that contains legal rules $\left(R_{\mathrm{L}}\right)$ and qualification rules $\left(R_{\mathrm{Q}}\right)$.

\subsection{Legal reasoning with arguments}

Whereas evidential arguments are used to establish the facts of a case, the next step is to determine the legal conclusions that can be based on these facts. First, the real-world facts have to be qualified as legal facts or, in other words, the factual stories have to be interpreted as legal stories on which a conviction can be based. Here the qualification rules of a legal theory $L T$ (specified in $R_{\mathrm{Q}}$ ) are guiding. Then these legal stories lead to a legal conclusion (on the basis of the legal rules in $R_{\mathrm{L}}$ ), which leads to the relevant legal consequences.

In the legal theory $L T$, rules are modelled in the same way as in the evidential and causal theories, that is, with a connective $\Rightarrow$ that satisfies defeasible modus ponens. We add two kinds of generalizations (or rules) to our formalisms: first the legal rules in $R_{\mathrm{L}}$ that warrant the step from legally relevant operative facts to a legal conclusion. An example is the Dutch legal rule that someone can be sentenced for murder, when he has killed someone, intentionally and with premeditation: $r_{\mathrm{L} 1}: x$ killed $y \wedge x$ intended to kill $y \wedge x$ killing $y$ was premeditated $\Rightarrow\llcorner x$ has murdered $y$.

The second additional kind of generalization is those that allow the qualification of a fact under a legally relevant term. An example of such a qualification rule is that if there are signs of the careful preparation of the murder weapon, then the killing was premeditated, viz. $r_{\mathrm{q} 1}: x$ prepared the murder weapon $\Rightarrow_{\mathrm{Q}} x$ killing $y$ was premeditated. Qualification rules may be based on, for example, linguistic conventions but they are often also warranted by jurisprudence.

The argumentative reasoning with qualification and legal rules (in the form of legal arguments) is modelled along the lines of existing work on this subject ([12], [13], [22]). We specifically base our formal model of arguments on [21].

Legal arguments can be built by taking elements from a story $S \in$ Stories $(C T)$ and rules from $R_{\mathrm{L}}$ as premises and chaining applications of defeasible modus ponens into tree-structured arguments. As an example, take the argument $A_{2}$ below, which says that if one person shoots another person and this causes the victim's death, then this counts as a killing (in the legal sense as required by one of the conditions of $r_{\mathrm{L} 1}$ ).
1. Francis shot Kevin
2. $r_{\mathrm{c} 1}$ : Francis shot Kevin $\Rightarrow \mathrm{c}$ Kevin died $\left(\right.$ story $\left.S_{1}\right)$
3. Kevin died
4. $x$ shoots $y \wedge r_{\mathrm{cl}} \wedge y$ dies $\Rightarrow_{\mathrm{Q}} x$ killed $y$
$\left(\right.$ story $\left.S_{1}\right)$
$\left(\right.$ story $\left.S_{1}\right)$
$\left(R_{\mathrm{Q}}\right)$
5. Francis killed Kevin

This argument can be extended to form an argument that Francis murdered Kevin, on the basis of the rule $r_{\mathrm{L} 1}$. This requires an argument for premeditation (for instance based on the qualification rule $r_{\mathrm{q} 1}$ ) and one for intention to kill.

Like in the evidential argumentation theory, counterarguments may be given to legal arguments. One may argue, for example, that if the a weapon was prepared not for killing someone but rather for a sporting activity, the preparation of the weapon does not count as premeditation; this would then undercut any inference based on the qualification rule $r_{\mathrm{q} 1}$. 


\subsection{Legal story schemes}

In purely argumentative approaches to legal reasoning (e.g. [1], [22]), the operative legal facts in the case are only considered as conditions of legal rules. However, we argue that they also serve another role, namely as a legal story scheme that provides a legally coherent perspective on a case. This is analogous to factual stories and story schemes, which provide a factually coherent perspective on a case.

A legal story scheme is a cluster of legally qualified states and events. In Dutch law, the definition of an offense in the criminal code can be used to extract a legal story scheme. An example of a legal story scheme is that for murder (in the legal sense):

$\{x$ killed $y, x$ intended to kill $y, x$ killing $y$ was premeditated $\}$.

Each element of the legal story scheme is one of the conditions of the legal rule for murder $r_{\mathrm{L} 1}$. In general, a legal story scheme $G_{\mathrm{L}}$ is a set of literal schemes. A legal story (e.g. an indictment) is then a specific instantiation of these literal schemes (e.g. \{Francis killed Kevin, Francis intended to kill Kevin, Francis killing Kevin was premeditated\}).

Legal story schemes are similar to factual story schemes in that they contain general event types, the relevant operative legal facts. We have made a slight distinction however: whereas factual story schemes are sequences, legal story schemes are sets, hence have no explicit ordering. Our reason for this modelling difference is that legal story schemes usually do not need an explicit notion of chronology, whereas factual story schemes do. A further difference between factual and legal stories is that legal stories do not normally contain causal rules of the form $c \Rightarrow \mathrm{c} e$; such logical rules are not needed in the legal theory $L T$, because prediction and abduction are not performed in $L T$ (recall that in $C T$ causal rules can be used to predict story consequences or to abduce possible causes). When some causal connection is an element of a legal condition (as for instance in criminally negligent manslaughter), however, that element will have to be made explicit in the legal story scheme.

\subsection{Connecting evidential and legal reasoning}

Given a legal story scheme and a story about the facts, the story somehow has to match the scheme, that is, the events in the factual story in some way have to correspond to the operative legal facts in the legal story. This correspondence is defined using arguments based on rules from $R_{\mathrm{Q}}$. Specifically, given a story $S$ and a legal story scheme $G_{\mathrm{L}}$, an element $s \in S$ corresponds to an element $g \in G_{\mathrm{L}}$ iff there is a justified argument $A \in A r g s_{\mathrm{L}}$ such that $\mathrm{s}$ is a premise of $A$ and a ground instance of $g$ is a conclusion of $A$. As an example, consider the subsequence [Francis shot Kevin, Francis shot Kevin $\Rightarrow c$ Kevin died, Kevin died] from story $S_{1}$. This part of $S_{1}$ corresponds to the legal story scheme for murder, because there is an argument $\left(A_{2}\right)$ which has these events as its premises and a ground instance of the legal story scheme for murder (Francis killed Kevin) as its conclusion.

Using the correspondence relation, we can define the legal completeness of a story. We say that a story $S$ completes a legal story scheme $G_{\mathrm{L}}$ iff for every element $g \in G$, there is some $s \in S$ such that $s$ is a ground instance of $g$. This legal completeness is similar to the factual completeness we defined in section 3.1: if a story completes a factual story scheme it is (causally) coherent and if a story completes a legal story scheme it is legally coherent. In a case, a story need not necessarily complete a factual story scheme (i.e. it need not be causally coherent); one can be sentenced for murder even if there is no clear motive. Legal story schemes, however, need to be completed (in the end), as otherwise we cannot say that the facts of the case (the story) support the indictment (the legal story based on the legal story scheme). This corresponds to the idea that a legal rule can only be applied if all its conditions are fulfilled. In criminal law, where the role of the legality or nulla poena principle is strong, this requirement is especially important.

As a result of the requirement that a legal story scheme be completed, there is a clear downward influence from the legal layer to the factual layer: facts that correspond to the legal story automatically become explananda. If a factual story wants to be considered as a serious candidate it has to be legally coherent; in order to be legally coherent the story the facts that correspond to the legal story scheme have to follow from the factual story.

Notice that the above definition of correspondence is similar to that of evidential support (section 3.3). In the case of correspondence, we connect the facts in the story to their legal interpretation (i.e. the legal story based on the scheme) by supporting the legal story with arguments based on the facts, whereas in the case of evidential support, we connect the evidence to their factual interpretation by supporting the factual story with arguments based on evidence. This similarity means that we can have legal gaps, elements of the legal story which are not supported by a fact from the factual story.

As said, in our approach factual stories may have evidential gaps. A legal story, however, cannot have such gaps at the end of an investigative process - as we just argued the factual story always needs to complete a legal story scheme. This leads to an interesting phenomenon: since gaps cannot occur at the legal level, gaps are pushed down from the legal level to the factual level. For instance, as murder requires premeditation, the legal story about the case will certainly have to contain premeditation, as otherwise the legal consequence of punishability for murder will not follow. The legal story will have to correspond to a factual concretisation of premeditation in the factual story, e.g., in the form of preparatory activities. However, it can happen that these preparatory activities are an evidential gap, i.e., remain unproven, and are only considered to exist as a result of the gap filling effects of stories. This implies that formally there is no legal gap, as the factual story does specify events corresponding to premeditation, while materially there is no direct support for the legal element of premeditation.

\subsection{Legal shifts and legal tunnel vision}

The legal perspective on the facts of a case drives not only what happens in court but also what occurs in the pre-trial investigative phase. In the Wamel case, it was quite clear that the factual perspective was murder (in the everyday, non-legal sense of the term): when a small-time (and perhaps not so small-time) criminal is found between barns, killed by bullet shots, the conclusion that we are dealing with a murder case is quickly made. Perhaps there is a brief consideration of the possibility of suicide, but the nature of the wounds may swiftly exclude that.

Initially the investigation will normally then be aimed at finding the killer. This is both required by what is expected in a factual story scheme for murder and in the corresponding legal murder scheme. When the killer is found (or at least someone who is seriously suspected of being the killer), the legal perspective becomes more prominent. The aim becomes to prove by evidence that the suspect is the killer ( $x$ killed $y$ in the legal story scheme for 
murder). The factual story scheme (which can include the killer, the circumstances of the killing, the murder weapon, the motive, and perhaps several other more factually oriented elements) remains relevant, but it becomes coloured by the legal perspective that is provided by the legal story scheme. For instance, the amount of detail of circumstances that need to be proven is steered by what is needed to complete the case in the legal sense.

This legal perspective that is leading a process of criminal investigation and decision making is modelled as a subset $L P$ of the legal story schemes $L S S$. LP is not a singleton but a set, as there can be more than one type of offense that is at issue. There is also the possibility of an empty perspective, when as yet it is unclear what kind of crime we are dealing with.

The explication of the legal perspective on a case allows the modelling of a legal shift. Here the Wamel case can serve as an illustration. In that case, the investigative process must have been somewhat frustrating. Already quite early Francis became a prime suspect. But notwithstanding much efforts put into questioning witnesses and other forms of information gathering, no clear proof was found that pinpointed Francis to the scene of the crime, let alone as the killer.

This must have resulted in a legal shift at some point. The case turned from a clear murder case (in the legal sense, with a murderer convicted) into one in which no murderer would be convicted. Instead, the case became a case of accomplicity to murder. The shift must have felt as a defeat, hard to accept. In fact, the bullet was for long not really bitten, as shown by the prosecution's charge (first instance) that Francis was the murderer, or else an accomplice. The murder charge was not repeated in second instance, completing the legal shift.

In a formal model of such a legal shift, the legal perspective $L P$ changes to a different legal perspective $L P$ '. Legal story schemes can be added - when a new kind of criminal offense is becoming part of the investigation - or removed - when some kind of offense is no longer considered relevant.

In the Wamel case, the shift is provoked by the failure of finding proof for a key element of the legal story scheme of murder. As a result, an accomplicity to murder scheme becomes the legal perspective. The legal murder scheme $\{x$ killed $y, x$ intended to kill $y, x$ killing $y$ was premeditated $\}$ that was an element of the legal perspective $L P$ is then replaced by an accomplicity to murder scheme $\{y$ is killed, the killing of $y$ was intentional, the killing of $y$ was premeditated, $x$ participated significantly in the killing of $y$ \}. In this scheme, it is unclear who actually killed the victim. Instead the significant participation in a killing becomes the criminally relevant criterion.

The Wamel case shows that the legal shift to the accomplicity perspective is not only needed to convict people that help a murderer, but also to convict someone who may well be the murderer but cannot be proven so.

It was already mentioned that Israëls has provided a careful analysis of alternative stories and their relative merits, thereby avoiding tunnel vision with respect to the facts. Our modelling of the legal perspective in a case shows that there can be a second kind of tunnel vision, namely by neglecting legal story schemes. For instance, an apparent murder case may in fact be a manslaughter case, or even one of negligent homicide. In this connection, we propose to speak of legal tunnel vision as opposed to factual tunnel vision. In the latter, one for instance neglects the consideration of other wrongdoers than one's favourite suspect (here: only considering Francis, and not also Sander), whereas in the former one focuses too much on finding proof for malice aforethought, whereas in fact it could be true that Francis wanted to frighten Kevin in order to become Uden's criminal alpha male, but in the ensuing fight accidentally shooting Kevin. Indeed such an accident might still legally count as murder, but the assumption of malice aforethought can have led to ignoring the possibility of an accident.

\section{DISCUSSION AND RELATED RESEARCH}

Our extended hybrid model is not the first that models the full pathway from evidence to legal consequences. [27] speaks of the rule-evidence interface, with 'rule' meaning 'legal rule'. He argues that the two sides of the rule-evidence interface have logical similarities and dissimilarities. He provides visualization techniques - akin to Wigmore charts - showing the path from evidence via proven facts to legal consequences. A similarity is that he uses tree structures for the representation of both the path from evidence to facts ('evidence evaluation'), and from facts to legal consequences ('rule-based deductions'). A difference is that evidence evaluation uses plausibility schemas and plausibility connectives, whereas rule-based deductions are modelled in terms of truth-functional connectives (with three truth values).

[1] also discuss analytic methods for reasoning with evidence in which the role of law is made explicit. They emphasise that the (potential) ultimate probandum (i.e., the legal consequence) needs to be made explicit at an early step in the process of analysing the evidence (step 2, p. 117).

In AI \& Law, evidential reasoning has also been addressed to some extent. For instance, although [13] focuses on the role of legal rules, it also discusses the issue of proof (p. 105-106). In this approach, proof is based on epistemic reasons, which can derive from rules of evidence as they are recognized in some jurisdiction. In [14] the model of rules is extended to a theory of qualitative, reason-based comparison of alternatives. In light of this theory, the connection with evidence and proof, and how different accounts of the facts can be compared on the basis of how well they fit the evidence is also discussed.

[22] have extensively modeled the legal part of the pathway and they focus on the formal modeling of the assessment and resolution of conflicting arguments as they occur in the law. While evidential reasoning plays a less important role, it is shown how argumentation concerning the admissibility of evidence in a case with forged evidence can be formalized (p. 342). This is one example of a "downward" influence (i.e. legal restrictions on evidence) which was not shown in this paper. However, given that our legal theory $L T$ is directly based on [21]'s extended version of [22], we think that the admissibility example can be rendered in our hybrid framework fairly easily.

The above related research is all mainly argumentative in style, as opposed to our hybrid proposal in which also the role of holistic, coherent narratives is made explicit. As is already extensively argued in [2], a hybrid theory in which arguments are combined with stories is best for reasoning about the evidence and the facts. In our opinion, including stories and (legal) story schemes also improves the model for legal reasoning. One case in point is the way in which legal gaps may be filled using facts which are themselves evidential gaps. Purely argumentative approaches, which do not have stories in the factual, middle layer, have no tools for filling such evidential gaps, that is, they miss the gap- 
filling function stories fulfil. In such argumentative approaches, the line of reasoning would go from the evidence all the way up to the operative legal facts (conditions of legal rules) and as a result of this, any legal fact that is not supported by evidence cannot be considered proven on the basis of the evidence.

Other relevant research which models the full pathway is the more narrative-oriented work by [18]. They show how coherent (factual) stories that explain the evidence can be matched to "verdict categories", which are essentially legal story schemes. The ideas provided in this work have been a great influence on the hybrid theory. A major difference though is that the authors of [18] approach evidential and legal reasoning from a psychological, descriptive stance whilst we also claim to have more normative aims (in [7], for example, we propose a list of pitfalls one should avoid when drafting a rational and intelligible verdict in a case). Furthermore, [18] does not fully formally specify the connection between the evidence, the facts and the law.

In our extended hybrid theory of inference to the best legal explanation, we reason at different layers of abstraction: a factual story is essentially the same as a legal story, but abstracts from the legal qualifications. One influential way of IBE with different layers is [9]'s abductive model, which models a hierarchy of causal networks. The causal networks in different layers are connected to each other using abstraction axioms (e.g. "swine flu is a type of influenza"). High-level concepts are then abductively explained by concepts lower in the hierarchy through abstraction axioms (e.g. if we observe influenza this can be swine flu or bird $f(u)$. These lower-level concepts can then be abductively explained through the usual causal rules. In theory, one could model the legal consequences as explananda at the highest level of abstraction and abductively infer the facts. For example, if we take the explanandum Francis killed Kevin, we can abductively infer the facts Francis shot Kevin, Francis shot Kevin $\Rightarrow_{c}$ Kevin died, Kevin died using $r_{\mathrm{q} 1}$. Thus, we could have a mainly story-based, causalabductive theory of legal reasoning.

Both [9] and [18] provide a story-based perspective on (evidential and legal) reasoning. Again, in [2] it was already argued why a hybrid theory which incorporates arguments is preferred to such purely narrative approaches. Briefly, the argumentative machinery (e.g. arguments and counterarguments, argumentation schemes and critical questions) provide a natural model of aspects of both evidential and legal reasoning.

\section{CONCLUSION AND SUMMARY}

In the previous sections, we have extended our hybrid narrativeargumentative approach to evidential reasoning in order to be able to address reasoning towards the legal consequences that can be attached to a case. Whereas before the hybrid theory was limited to evidential reasoning and stopped at the facts, we now can complete a line of legal reasoning up until the legal decision. We have introduced the notion of a legal story scheme, thereby allowing the explication of the legal perspective taken in a case analysis (formalized as an extended hybrid theory). The legal perspective is relevant in a case analysis as it steers the investigative and decision-making process. In fact, there is a legal analogue of the familiar factual tunnel vision that we dubbed legal tunnel vision: when one too strongly focuses on the evidential needs to complete a case in terms of one kind of legal perspective, better alternatives may be pushed out.

Our proposal can be summarized as follows.
1. In our approach, the hybrid narrative-argumentative style of modeling is used for the full pathway of reasoning from evidence via facts to legal consequences. As a result, we use our hybrid approach for a unifying view on both evidential and legal reasoning.

2. In comparison with purely argumentative approaches, our use of story schemes (both at the evidential and at the legal step) allows for the explicit and natural modeling of a coherent, holistic perspective on a case, as it underlies an investigative and decision making process.

3. At the factual level, story schemes show evidential gaps, showing where to strengthen investigative efforts, and point to alternatives, thereby preventing tunnel vision. Factual shifts occur when the perspective on the facts (in the form of a favorite factual story scheme) is changed, by adding and/or removing story schemes that steer the evidence marshaling process.

4. At the legal level, something similar occurs. A legal story scheme provides a holistic perspective that steers the process of reasoning towards legal consequences. The risk of legal tunnel vision can be reduced by the consideration of alternative legal story schemes. Legal shifts can be modeled in the form of the addition or removal of a legal story scheme.

5. But at the legal level, there is no analog of evidential gaps: every element of a legal story needs to be covered. This corresponds to the fact that every condition of a criminal legal rule needs to be satisfied in order for the legal consequence to follow (cf. the legality principle that is dominant in criminal law).

6. In our approach, it is made explicit that legal gaps can be pushed down to the factual level. Every legal element that is required to complete a legal story is matched to an element in the factual story. Whether all elements of the factual story are considered to be proven, allowing the possibility of an evidential gap, is then determined at the evidential level.

An issue with our hybrid narrative-argumentative approach is that in its present form we do not provide a deep connection between stories and arguments whereas there seems to be one. For instance, one reasonable story provides a counterargument to another. In our earlier work, we have suggested that arguments and stories are a kind of 'communicating vessels'. In the present setting of legal story schemes, there is the related issue how the legal conditions of a valid rule are connected to a legal story scheme. One perspective (underlying this paper) is that the legal conditions provide the atomistic building blocks for a legal story scheme. As a result of the holistic character of legal story schemes, they can be used to establish the legal coherence of a case analysis.

\section{REFERENCES}

[1] Anderson, T.J., Schum, D.A., and Twining, W.L. 2005. Analysis of Evidence, 2nd edition. Cambridge University Press, Cambridge.

[2] Bench-Capon, T. and Sartor, G. 2001. A Quantitative Approach to Theory Coherence. In B. Verheij, A.R. Lodder, R.P. Loui, A. Muntjewerff (Eds.), JURIX 2001: The 14th annual conference, 53-62. IOS Press, Amsterdam.

[3] Bex, F.J. 2011. Arguments, Stories and Criminal Evidence: A Formal Hybrid Theory. Springer, Dordrecht. 
[4] Bex, F.J., van Koppen, P.J., Prakken, H., and Verheij, B. 2010. A Hybrid Formal Theory of Arguments, Stories and Criminal Evidence. Artificial Intelligence and Law 18:2, 123-152.

[5] Bex, F.J., Prakken, H., Reed, C., and Walton, D.N. 2003. Towards a formal account of reasoning about evidence: argumentation schemes and generalisations. Artificial Intelligence and Law 11, 125-165.

[6] Bex, F.J., Prakken, H. and Verheij, B. 2007. Formalising Argumentative Story-based Analysis of Evidence. The 11th International Conference on Artificial Intelligence and Law, 1-10. ACM, New York.

[7] Bex, F.J., and Verheij, B. (2009). Het onderbouwen van een feitelijk oordeel in een strafzaak: methode, casus, aanbevelingen (Grounding a judgement about the facts in a criminal case: method, case, recommendations). In P.J. van Koppen, H. Merkelbach, M. Jelicic and J.W. de Keijser (Eds.), Reizen met mijn Rechter: Psychologie van het Recht (pp. 935-952). Kluwer, Deventer.

[8] Bex, F.J. and Walton, D. 2010. Burdens and Standards of Proof for Inference to the Best Explanation. In R. Winkels

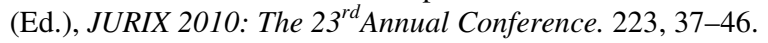
IOS Press, Amsterdam.

[9] Console, L., and Dupré, D.T. 1994. Abductive reasoning with abstraction axioms. Lecture Notes In Computer Science 810, 98-112.

[10] Console, L. and Torasso, P. 1991. A spectrum of logical definitions of model-based diagnosis. Computational Intelligence 7: 133-141.

[11] Dung, P.M. 1995. On the acceptability of arguments and its fundamental role in nonmonotonic reasoning, logic programming, and n-person games. Artificial Intelligence 77, 321-357.

[12] Gordon, T.F. 1987. Some Problems with Prolog as a Knowledge Representation Language for Legal Expert Systems. In C. Arnold (Ed.), Yearbook of Law, Computers and Technology, 52-67. Leicester Polytechnic Press, Leicester.

[13] Hage, J.C. 1997. Reasoning with Rules. An Essay on Legal Reasoning and Its Underlying Logic. Kluwer, Dordrecht.

[14] Hage, J.C. 2001. Formalising Legal Coherence. Proceedings of the 8th International Conference on Artificial Intelligence and Law, 22-31. ACM, New York.

[15] Hage, J.C. 2005. Studies in Legal Logic. Springer, Dordrecht.

[16] Israëls, H. 2006. Moord in Wamel (Murder in Wamel). Boom Juridische Uitgevers, The Hague.
[17] Keppens J. and Schäfer B. 2006. Knowledge based crime scenario modelling. Expert Systems and Applications 30, 203-222.

[18] McCarty, L.T. 1997. Some Arguments About Legal Arguments. The 6th International Conference on Artificial Intelligence and Law, 215-224. ACM, New York.

[19] Pennington, N., and Hastie, R. 1993. Reasoning in explanation-based decision making. Cognition 49:1-2, 123 163.

[20] Pollock, J. L. 1995. Cognitive Carpentry: A Blueprint for How to Build a Person. MIT Press, Cambridge.

[21] Prakken, H. 2010. An abstract framework for argumentation with structured arguments. Argument and Computation 1, 93-124.

[22] Prakken, H. and Sartor, G. 1996. A Dialectical Model of Assessing Conflicting Arguments in Legal Reasoning. Artificial Intelligence and Law 4: 331-368.

[23] Prakken H. and Renooij S. 2001. Reconstructing causal reasoning about evidence: a case study. In B. Verheij, A.R. Lodder, R.P. Loui, A. Muntjewerff (Eds.), JURIX 2001: The 14th annual conference, 160-169. IOS Press, Amsterdam.

[24] Schank, R.C. 1986. Explanations Patterns: Understanding Mechanically and Creatively. Lawrence Erlbaum, New Jersey.

[25] Thagard, P. 2004. Causal Inference in Legal Decision Making: Explanatory Coherence vs. Bayesian Networks. Applied Artificial Intelligence 18:3, 231-249.

[26] Verheij, B. 2005. Virtual Arguments. On the Design of Argument Assistants for Lawyers and Other Arguers. T.M.C. Asser Press, The Hague.

[27] Walker, V.R. 2007. Visualizing the Dynamics around the Rule/Evidence Interface in Legal Reasoning. Law, Probability and Risk 6: 5-22.

[28] Wagenaar, W.A., Koppen, P.J. van, and Crombag, H.F.M. 1993. Anchored Narratives: The Psychology of Criminal Evidence. St. Martin's Press, New York.

[29] Walton, D.N., Reed, C.A. and Macagno, F. 2008. Argumentation Schemes. Cambridge University Press, Cambridge.

[30] Walton, D.N., and Schafer, B. 2006. Arthur, George and the mystery of the missing motive: towards a theory of evidentiary reasoning about motives. International Commentary on Evidence 4(2):1-47. 